\title{
Title: Role of X-ray Scattering Techniques in Understanding the Collagen Structure of Leather
}

\begin{abstract}
This review focuses on the SAXS and WAXS analysis of the collagen structure of leather, from pioneering works through to recent developments. X-ray scattering can be used to investigate the structural features of fibrillar collagen in the leather matrix, including axial periodicity, fibril diameter, chemical fixation, hydration and fibrillar orientation. Key properties of leather, such as appearance, handle, strength, hydrothermal stability and resistance to putrefaction, are closely linked to these parameters, thereby underlining the importance of $X$ ray scattering to the study of leather. Recent research on the molecular level changes in collagen structure at different stages of leather processing with a range of treatments is also summarized. We will highlight how studies using X-ray scattering techniques have provided significant insights into tanning processes which could be used to improve leather properties and optimize/reduce the use of tanning agents such as chromium (III) in the industry. The review will also describe the details of collagen structure and tanning mechanisms that are yet to be determined by X-ray scattering, as well as future advancements of this technique and what it can accomplish for the leather industry.
\end{abstract}

\section{Introduction}

Collagen, a major constituent in many hierarchically organized biological materials is the main structural component in animal hides and skins. 1 In the past 15 years, many researchers have devoted themselves to the investigation of collagen structure of leather via X-ray scattering methods. Small-angle X-ray scattering (SAXS) can used to study the properties of macromolecular structures ranging from 1-100 nanometres, while wide-angle $X$-ray scattering (WAXS) focuses on the molecular level details ranging from a few nanometres to sub-nanometre scales. 2 The structure of animal skins and hides (before or after processing into leather) over a range of length scales from the macroscopic to the molecular, is known to impact on its performance and properties. The intermolecular later al packing, amorphous polypeptide features and helical rise per residue of collagen in leather can be deduced from the peaks in the WAXS intensity spectrum. $\underline{3}$ Through analysis of the size and position of diffraction peaks in the SAXS intensity profile, information has been obtained on the axial periodicity, chemical fixation and cross-linking, hydration, and fibrillar size and orientation of the collagen molecules in leathers. $\underline{4}$

Basic chromium sulphate (chrome/BCS) is the most widely used tanning agent, converting putrescible skin into non-putrescible leather with improved physical and hydrothermal properties. $\underline{\mathbf{5}}$ The chrome tanning reaction occurs by the complexation of chromium(III) molecular ions at the ionized carboxyl groups of aspartic and glutamic acid residues of collagen, however detailed knowledge of this reaction remains elusive. $\underline{3 d}, \underline{6}$ 
From X-ray scattering studies of leather, we have gained insights into tanning processes which could help reduce the amount of chrome used in industry. Changes in the chemical fixation, hydration and $D$-periodicity of collagen were obtained from SAXS analysis during different stages of leather tanning and gave further understanding of the tanning mechanism. $4 \underline{p}$ Studies have also investigated the relationships between leather properties (e. g., strength, $\underline{\mathbf{4 b}}-\underline{\mathbf{4 e}}$ pebbling $\underline{4}$ - a characteristic pattern in deer leather and looseness $\underline{\mathbf{4 m}}$ ) and the structure of collagen, which can help to produce leather of better quality.

In this mini-review we will summarize the X-ray scattering studies of the collagen structure in leather, and its applications in the leather industry. We will explain how SAXS and WAXS are used to probe the collagen structure of leather and the relation between features of collagen structure and its physical and hydrothermal properties. Improvements and changes can be made to the tanning processes using this information to make quality leather sustainably.

\section{Collagen Structure}

The main component of animal skins, tendons and bones is type I collagen, which is the most abundant of the 28 types of collagen which have been identified so far. 1 The mechanical properties of fibrous collagen come from its hierarchical structure of collagen molecules, fibrils and fibers (Scheme 1). $\mathbf{7}$ All types of collagen feature tropocollagen, a righthanded triple helical structure of three polypeptide chains with repeating s equence of Gly$X-Y$ where $X$ and $Y$ are arbitrary amino acids, commonly proline and hydroxyproline. 1 With glycine present in the core of the triple helix, $X$ and $Y$ are exposed to the surface of the collagen molecule.1a The side chains of $X$ and $Y$ are involved in intermolecular interactions such as hydrogen bonding, which help stabilize the collagen molecule and form fibrils.1b The fibril is a critical component of the hierarchical structure of fibrous collagen, where the structural organization within the fibril contributes toward the mechanical properties of the biological material. $\underline{\mathbf{1 b}}$ X-ray diffraction studies on collagen molecular packing within a single fibril have been performed since the 1960 s and were observed to be composed of tropocollagen molecules in a quarter-staggered pattern. $\underline{\mathbf{8}}$ Known as the HodgePetruska model, it describes the axial staggering as well as the lateral packing of tropocollagen molecules. The periodic gap and overlap regions between each two consecutive collagen molecules in the axial staggering give fibrillar collagen an observable banding feature called the $D$-period or $D$-spacing. $\underline{\mathbf{8}}, \underline{9}$ The $D$-period of type I collagen in skin or leather, usually about $65.5 \mathrm{~nm}, \underline{\mathbf{2 d}}, \underline{\mathbf{1 0}}$ is sensitive to various structural modifications of collagen, such as chemical cross-links, $\mathrm{pH}$, hydration level and mechanical strains. $\underline{9}$ 


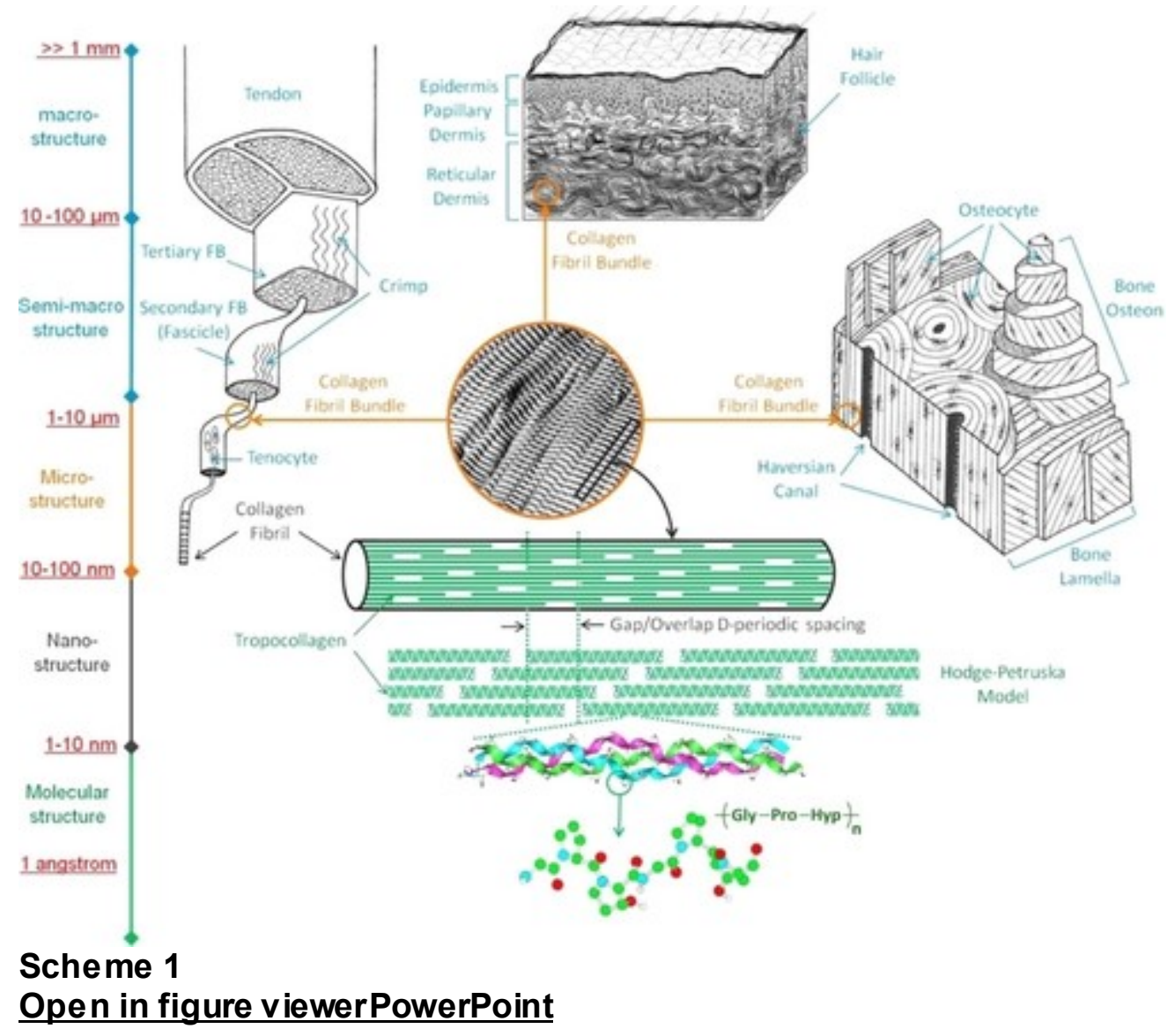

The packing of the collagen molecule in tendon, skin and bone. Reproduced with permission from Ref. [7]. Copyright 2012, American Chemical Society.

\section{$3 \mathrm{X}$-ray scattering techniques}

Early studies on the $D$-periodicity and size of collagen fibrils in skin and leather mainly relied on microscopic techniques.11 However, because of the complicated pattern of fibril arrangement as well as the sensitivity of the $D$-period to condition changes and chemical treatments, it is hard to attain accurate values for the parameters of collagen fibrils via imaging. Most studies of collagen using these methods have significant errors for these parameters of around a few nanometres.11 Recently, researchers have explored a less destructive way of measuring structural factors of collagen in leathers in their native state by applying X-ray scattering techniques. $\underline{\mathbf{3}}, \underline{\mathbf{1 2}}$

X-ray scattering techniques are widely used for the structural analysis of crystals and amorphous materials.13 Basically, when an incident X-ray beam lands on a material, the electrons within the material are excited by the electromagnetic field. When the excited electrons relax, the $X$-ray radiation is released in a different direction to the incident beam. $A$ scattering pattern is produced from the superposition of the X-rays scattered by the substrate.2a If the molecules in the material are regularly packed, the periodic arrangement is reflected in the diffraction pattern.14 Therefore, the staggered periodicity and the fibril 
arrangement of collagen in leather can be studied by X-ray scattering to gain detailed information of its molecular and interfibrillar structures. The intensity of the scattering pattern is also informative, as intensity increases with increasing electron density contrast.2b Although biological materials such as skin collagen prior to tanning are mostly composed of light atoms only, tanning with mineral compounds involves binding with metal ions and thereby increases the intensity of the diffraction peaks (Bragg peaks). $\underline{\mathbf{3 b}}$

\section{Leather processing}

The major stages of leather processing usually consist of the following steps: liming, deliming, pickling, tanning, fatliquoring, dyeing and drying. The key step is tanning as this converts the skin to a material that is stable to putrefaction and has increased hydrothermal stability.15 Basic chromium sulphate (chrome) is the most commonly used tanning agent in the leather industry, due to the short processing time and high hydrothermal stability. $\underline{\mathbf{5 b}}, \underline{15}$ Figure 1 illustrates a proposed structural model of chromium(III) species and sulfate ions when reacting with collagen in leather.15 The reaction occurs between chromium(III) molecular ions and the ionized carboxyl groups of aspartic and glutamic acid residues of collagen (there may be preference for aspartate over glutamate). 16 With the presence of sulfate ions, the chromium molecular ions can be linked together, forming a linear $\mathrm{Cr}_{4}$ olated complex.15 The coordinated sulfate is not part of the ligand field of the chromium complex, but is needed for the creation of the supramolecular tanning matrix.5a However, the major disadvantage of chrome tanning is its (incorrectly) perceived environmental impact: wastewater from conventional chrome tanning contains some chromium due to $70-95 \%$ uptake.15, 17 The potential toxicity due to conversion to the toxic hexavalent state is almost certainly overstated, since it is reversed in a reducing environment, such as soil. 18 Nevertheless, this has led to researchers searching for alternatives or improvements to the chrome tanning process. 


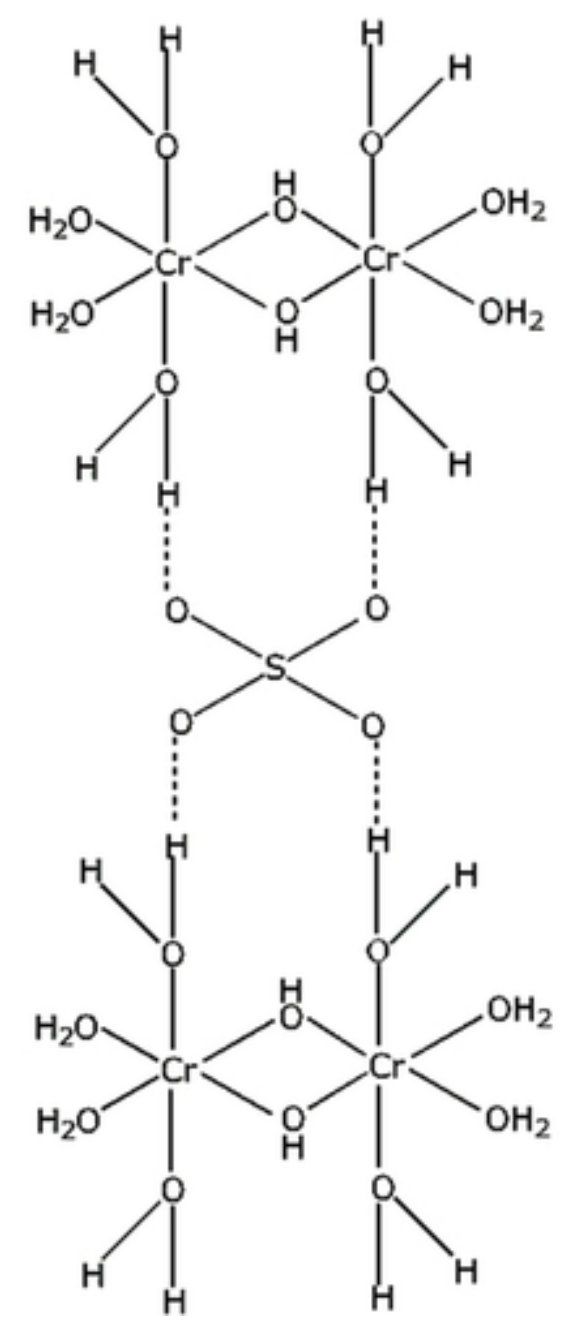

Figure 1

\section{Open in figure viewerPowerPoint}

Possible structural model of chromium (III) species and sulfate ions in leather. Reproduced with permission from Ref. [15]. Copyright 2009, The Royal Society of Chemistry.

Other tanning agents include vegetable tannins, aluminum, titanium, zirconium, oil, aldehydes and syntans (synthetic tanning agents). Vegetable tannins predominantly hydrogen bond to collagen via their phenolic hydroxyl groups, but the so-called condensed tannins based on the flavonoid fused ring structure may also partially fix covalently. $\underline{\mathbf{b}}, \underline{15}$ Aluminum(III) only weakly interacts with collagen, forming electrovalent interactions with the carbonyl groups. $\underline{\mathbf{5 b}}, \underline{\mathbf{1 5}}$ Chains of titanium(IV) molecular ions bridged by oxygen atoms, $(\mathrm{Ti}-\mathrm{O}-\mathrm{Ti})_{\mathrm{n}}$, also interact with collagen via electrovalent interactions. $\underline{\mathbf{5 b}}, \underline{15}$ Tetrameric zirconium(IV) units can polymerize to fix electrostatically and hydrogen bond to collagen via the hydroxyl groups. $\underline{\mathbf{b}}$ Aldehydes mainly react with collagen amino groups, the sulfonate groups of syntans also interact with the amino side-chains of collagen, while the nature of oil tanning is anomalous since it modifies the collagen structure without directly bonding to it. However, none of these tanning agents can produce the high shrinkage temperatures of chrome tanned leathers. $\underline{\mathbf{5 b}}, \underline{\mathbf{1 5}}$ 


\section{X-ray scattering studies of leather}

With the help of X-ray scattering techniques, insights into the structure of collagen in leather can be acquired and applied as a guide for modifying industrial leather tanning processes.

The aims of industrial tanning processes are to make leather products with better appearance, handle, strength, hydrothermal stability, resistance to putrefaction as well as a variety of other properties.15 Appearance and handle are dependent on the chemical properties of the reagents used in the leather tanning process.15 Leather strength is related to the orientation of the collagen fibrils. $\underline{\mathbf{4 c}}, \underline{\mathbf{4 f}} \mathrm{Hydrothermal} \mathrm{stability} \mathrm{is} \mathrm{the} \mathrm{resistance} \mathrm{of} \mathrm{the}$ material to wet heat, typically gauged by the shrinkage temperature of hydrated leather.15, 19 The definition of tanning is not related to hydrothermal stability but to resistance to putrefaction by microorganisms, although both are dependent upon the moisture content which creates the supramolecular matrix which in turn contributes to the stability of the collagen structure.15 All of these properties relate to the collagen structure of leather at the molecular level. However, in spite of the long history of leather tanning, the detailed mechanism of tanning and the role of collagen fibrillar structure on leather properties remain unclear. X-ray scattering techniques are generally separated into two categories: small- and wide- angle X-ray scattering (SAXS and WAXS). 2a SAXS usually refers to $X$-ray scattering at angles less than $5^{\circ}$ and is used to investigate structures ranging from one to hundreds of nanometres. $\underline{2}$ In contrast, WAXS generally covers scattering at angles up to $80^{\circ}$ and is used to resolve the subnanometre-sized molecular level details. $\underline{2 a}, \underline{2 d}, \underline{20}$

The regular arrangement of collagen molecules in skin and leather leads to a WAXS spectrum which features three peaks that relate to structural features of collagen (Figure 2). 3 c The first peak corresponds to the intermolecular lateral packing of the collagen molecules, the second represents the amorphous polypeptide features of fibrillar collagen, and the third is related to the helical rise per residue. $\underline{\mathbf{2 d}}, \underline{\mathbf{3}}, \underline{\mathbf{2 1}}$ 


\section{Dry Salted Bovine Hide}

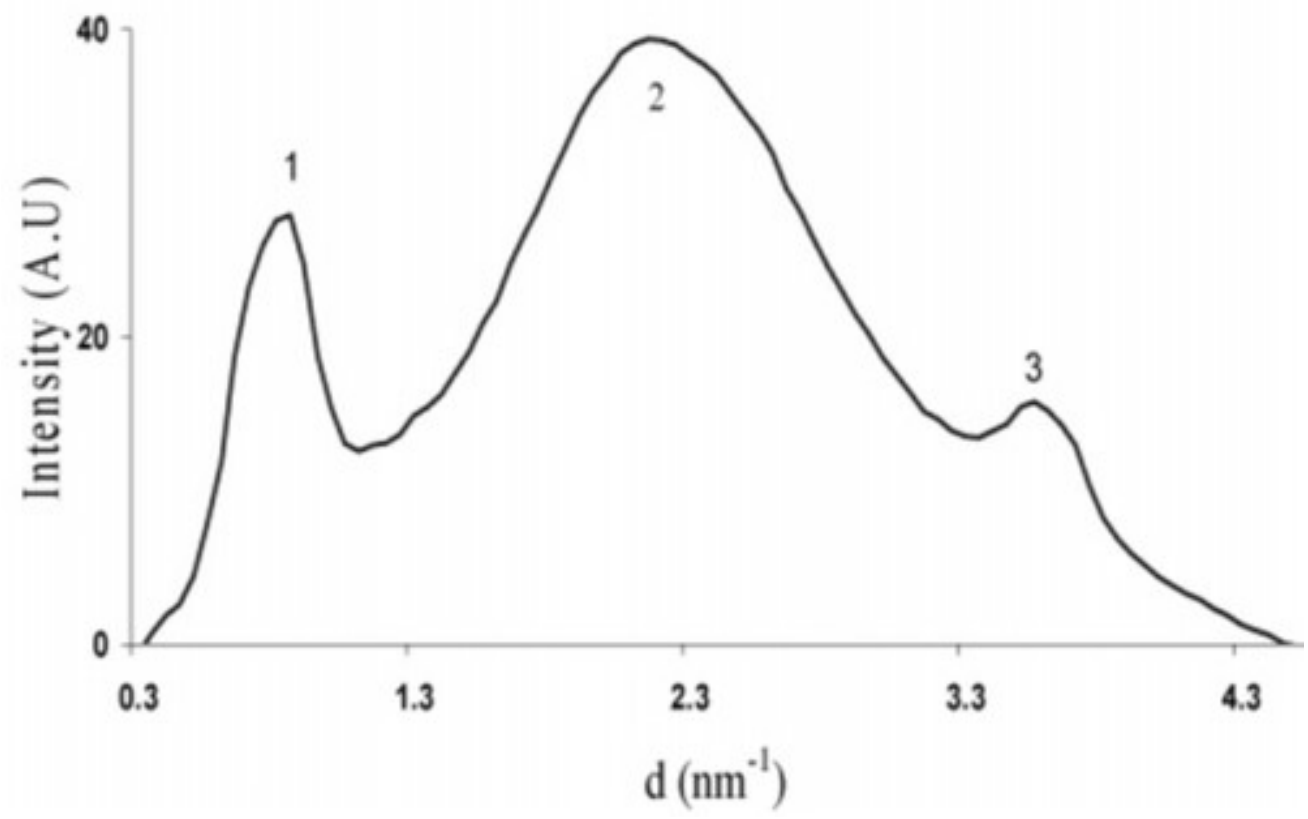

Figure 2

\section{Open in figure viewerPowerPoint}

Linear intensity profile of a dry salted bovine hide sample. Reproduced with permission from Ref. [3c]. Copyright 2006, American Chemical Society.

SAXS analyses of skins and leathers have resulted in characteristic diffraction patterns due to the long-range order of the collagen molecules, with repeating gap/overlap regions in a characteristic axial periodicity (Figure $\underline{3}$ ). $\underline{3 a}-\underline{3 c}, \underline{4 p}-\underline{4 s}$ The form factor oscillation pattern can also be detected in a SAXS spectrum which is attributed to the ordered arrangement of collagen fibrils. From these spectra, the $D$-period and fibril diameter can be calculated. The peak positions and intensities can also provide information on the collagen cross-linking and hydration, as well as the orientation of the collagen fibrils.
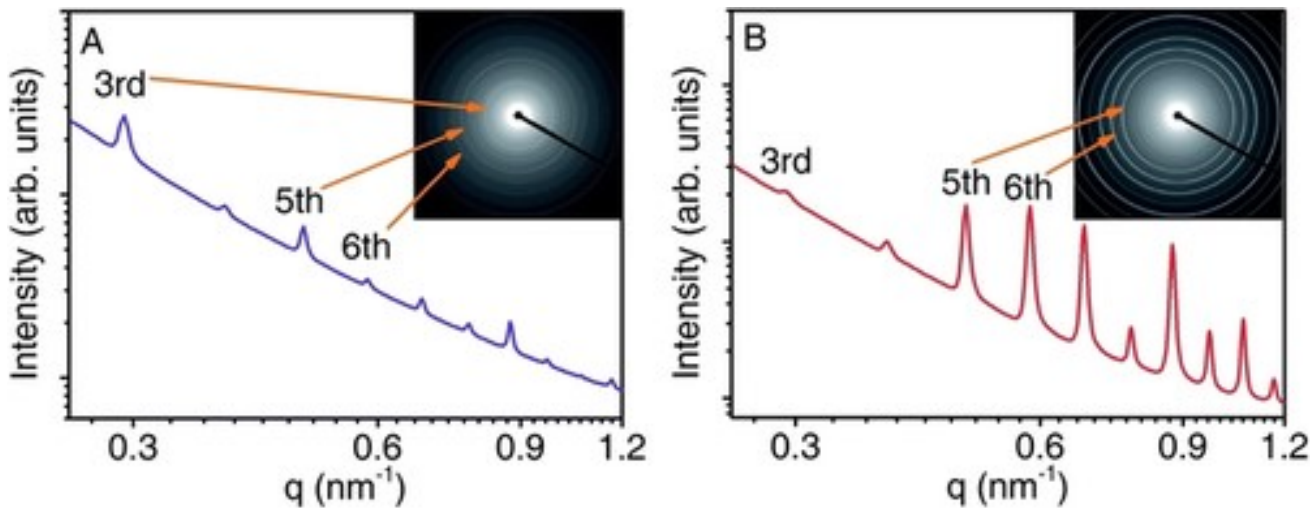

Figure 3

Open in figure viewerPowerPoint

SAXS diffraction patterns for a) pre-tanned and b) chrome tanned lambskins. Selected diffraction peaks corresponding to $q=2 \pi n / D$ where $n$ is the peak order and $D$ is the $D$-period, 
are labelled. Reproduced with permission from Ref. [4q]. Copyright 2017, The Royal Society of Chemistry.

An increase in the intensities of the Bragg peaks occurs in the SAXS spectrum upon chrome tanning. There are two possible causes of this: an increase in the overall long-range order of the collagen molecules and/or the chromium(III) ions present in the collagen structure enhancing the electron density contrast. $\underline{3 \mathbf{b}}, \underline{\mathbf{4}} \underline{\mathbf{p}} \underline{\mathbf{4 s}}$ Maxwell et al. noted that if the increase in peak intensities in the SAXS spectra of chromium treated bovine hide was predominantly caused by the enhanced electron density contrast due to chromium(III), then the binding of chromium(III) along the length of the collagen molecule must be relatively even to cause the same intensity distribution of the peaks compared to the untanned hide. If the binding of collagen is limited to particular amino acids, then these amino acids must have a relatively even distribution through the collagen molecule. $\underline{3 b}$

Our group has studied the changes in SAXS spectra after processing with three different tanning methods: BCS, zirconium sulfate (ZIR) and an aluminosilicate/poly(carboxylic acid) based tanning agent (ALS).4p No intensity changes were observed in the SAXS spectra between the pickled and pre-tanned skins. On tanning with BCS, ZIR or ALS, there was a significant increase in the intensities of the fifth - eleventh order peaks and a decrease in the third order peak. $\underline{4}$ The peak intensities of the ALS tanned leather were all lower than those of the BCS and ZIR tanned leather. This was attributed to the lower electron density of the $\mathrm{Al} / \mathrm{Si}$ of the aluminosilicates compared to the chromium and zirconium ions of BCS and

\section{ZIR.4p}

\subsection{WAXS}

\subsubsection{Inte rmolecular lateral packing}

The intermolecular lateral packing is the distance between collagen molecules in the lateral plane of the collagen fibril.21a The position of peaks in a SAXS or WAXS spectrum is directly related to the spacing of the repeating units in the collagenous substrate. Therefore, the intermolecular lateral packing distance in collagen can be calculated from their X-ray scattering pattern using Bragg's Law:

$$
2 d \sin \theta=n \lambda
$$

where $\theta$ is the angle between the wave vector of the incident plane wave and the lattice planes, $\lambda$ is the wavelength, and $n$ is the order of the reflection. The difference between the incident and scattered wave vectors is described as the momentum transfer vector $q$, where its magnitude can be calculated as: $\underline{22}$

$$
q=(4 \pi \sin \theta) / \lambda
$$

These two equations can be combined to give the lateral packing distance: $\underline{4 h}, \underline{4 p}-\underline{4 s}$

$$
d=2 \pi n / q
$$

An increase of around $0.1 \mathrm{~nm}$ was observed in the WAXS spectrum for the intermolecular lateral packing of hydrated collagen molecules in bovine hide after salting compared to untreated hide. $\underline{3 \mathrm{c}}$ Liming of hide was found to increase the intermolecular lateral packing 
distance by approximately $0.15 \mathrm{~nm}$ compared to untreated hide. $\underline{3 \mathrm{c}}$ Deliming was found to have no effect on the distance between collagen molecules, which is consistent with previous observations that liming permanently affects the structure of the collagen matrix. $\underline{3 c}, \underline{15}$

Increasing concentration of chrome has been observed to decrease the height of the peak corresponding to the intermolecular lateral packing. The intermolecular lateral packing distance slightly decreased after the addition of chrome and the full width half maxima (FWHM) of this peak increased, indicating a broader distribution. $\underline{\mathbf{3 b}}$ The decrease in the peak intensity at $0.89 \mathrm{~nm}$ appeared to be correlated with the reduction in order of the intermolecular packing distance. Another peak at $0.935 \mathrm{~nm}$, which was not present in the control sample, appeared with increasing chrome concentration, and lipid bilayer interactions with $\mathrm{Cr}^{3+}$ ions were suggested to be a possible cause of this peak. $\underline{3 \mathbf{b}}$

WAXS has been used to investigate the changes in collagen structure during tanning of pigskin collagen fibers with different concentrations of $\mathrm{Cr}^{3+} .3 \mathrm{~d}$ Peak 1, attributed to the intermolecular lateral packing, decreased with chrome tanning to the point where it almost disappeared. This provided evidence that within the microfibrils, the $\mathrm{Cr}^{3+}$ ions had penetrated the gap between collagen molecules. $\underline{\mathbf{3 d}}$

An increase in the intermolecular lateral packing distance was observed with increasing moisture for both stretched tanned leather and the non-stretched control, with the stretched leather showing a smaller increase. The grain and corium were not found to have significantly different intermolecular spacing. $\underline{\mathbf{4 h}}$

\subsubsection{Amorphous polypeptide features}

Amorphous scatter in a collagen sample results from denatured collagen (typically from natural saprophytic bacterial attack and subsequent chemical damage from soaking and liming) and other non-collagenous materials. $\underline{3 c}$ A ratio of collagen to amorphous content within a sample can be calculated by dividing the integrated intensity of the first peak (intermolecular lateral packing) by the second peak (amorphous scatter) of the WAXS spectrum. The ratio of collagen to amorphous material was found to increase when the untreated hide was salted and limed. $\underline{\mathbf{3 c}}$ The amorphous scatter of collagen samples is often considered in relation to other features, such as the axial rise per residue, to ensure that peak intensity changes in the WAXS spectrum are due to collagen structural changes rather than degradative changes.

\subsubsection{Helical rise per residue}

The helical rise per residue is the axial rise distance between amino acid residues along the helix axis. The helical rise per residue of bovine hide was approximately constant $(0.277-$ 
$0.279 \mathrm{~nm}$ ) through the different treatments of salting, liming and deliming, although the FWHM for this peak was observed to increase through the different stages of treatment. $\underline{3 c}$

WAXS of hide leather treated with varying concentrations of chrome showed a reduction in peak height and increase in FWHM for the axial rise per residue as the concentration of $\mathrm{Cr}_{2} \mathrm{O}_{3}$ increased. $\mathbf{3 b}$ This indicated that at the molecular level, there was a loss of order in the collagen molecules as the chromium salt concentration increased. The lack of a corresponding increase in the amorphous scatter suggests that the amount of degradation did not increase and, may have been due to the significant changes made to the helical backbone of collagen without completely damaging its structure. $3 \mathrm{~b}$ This would be consistent with the view that chrome tanning modifies the supramolecular matrix of hydrating water present in the natural state of skin.

Similarly, after the introduction of $\mathrm{Cr}^{3+}$ to pigskin collagen fibres, the peak corresponding to axial rise per residue decreased, without a concomitant increase in gelatinization. This was attributed to the complexation of $\mathrm{Cr}^{3+}$ with side-chain carboxyl groups of aspartic and glutamic acids of collagen, causing the structure of collagen molecules to become distorted. Furthermore, the diffraction intensity of $\mathrm{N}$ and $\mathrm{C}$ telopeptides decreased as the concentration of $\mathrm{Cr}^{3+}$ increased. This was believed to be due to the distortion of telopeptides caused by the deposition of $\mathrm{Cr}^{3+}$ ions since the telopeptide regions are as reactive as the helical regions to chrome tanning. $\underline{\mathbf{3 d}}$

While WAXS provides useful information on the collagen structure in leather, more recent studies have focused on SAXS to gain information on collagen modification by chemical fixation, fibril orientation, hydration, axial periodicity and fibril diameter.

\subsection{SAXS}

\subsubsection{Collagen modification by chemical fixation}

The relative intensity of the fifth to third order peak has been associated with the covalent bonding of chromium with collagen. Comparison of SAXS data of untanned skins and chrome-tanned leathers found a greater relative intensity of the fifth to third order peak for the tanned leathers. $\underline{4 r}$ In addition, the intensity of the third order peak initially decreased from a chromium concentration of $0 \%$ to $1.8 \%$ and disappeared at $3.7 \%$, followed by reappearance at $6.3 \%$ (Figure $\underline{4}$ ). 4 r This suggested there was initially covalent bonding of chromium with glutamic and aspartic acid residues, resulting in a gradual decrease of the third order peak and an increase in intensity in the other peaks, until all glutamic and aspartic acid residues are exhausted. Non-covalent interactions, such as hydrogen bonds between the aquo ligands and hydroxo bridges of the chromium complexes and the charged amino acid residues, then resulted in a layer of chromium species deposited around the collagen 
molecules. 4 r Such an outcome is possible when very high offers of chromium salt are applied under reactive conditions, so that a significant part of the deposited chromium does not contribute to the hydrothermal stability. This would support the development of chrome tanning technologies that focus on reducing chrome usage by enhancing its uniform penetration through the leather matrix using alternative carrier mediums. $\underline{\mathbf{2 3}}$

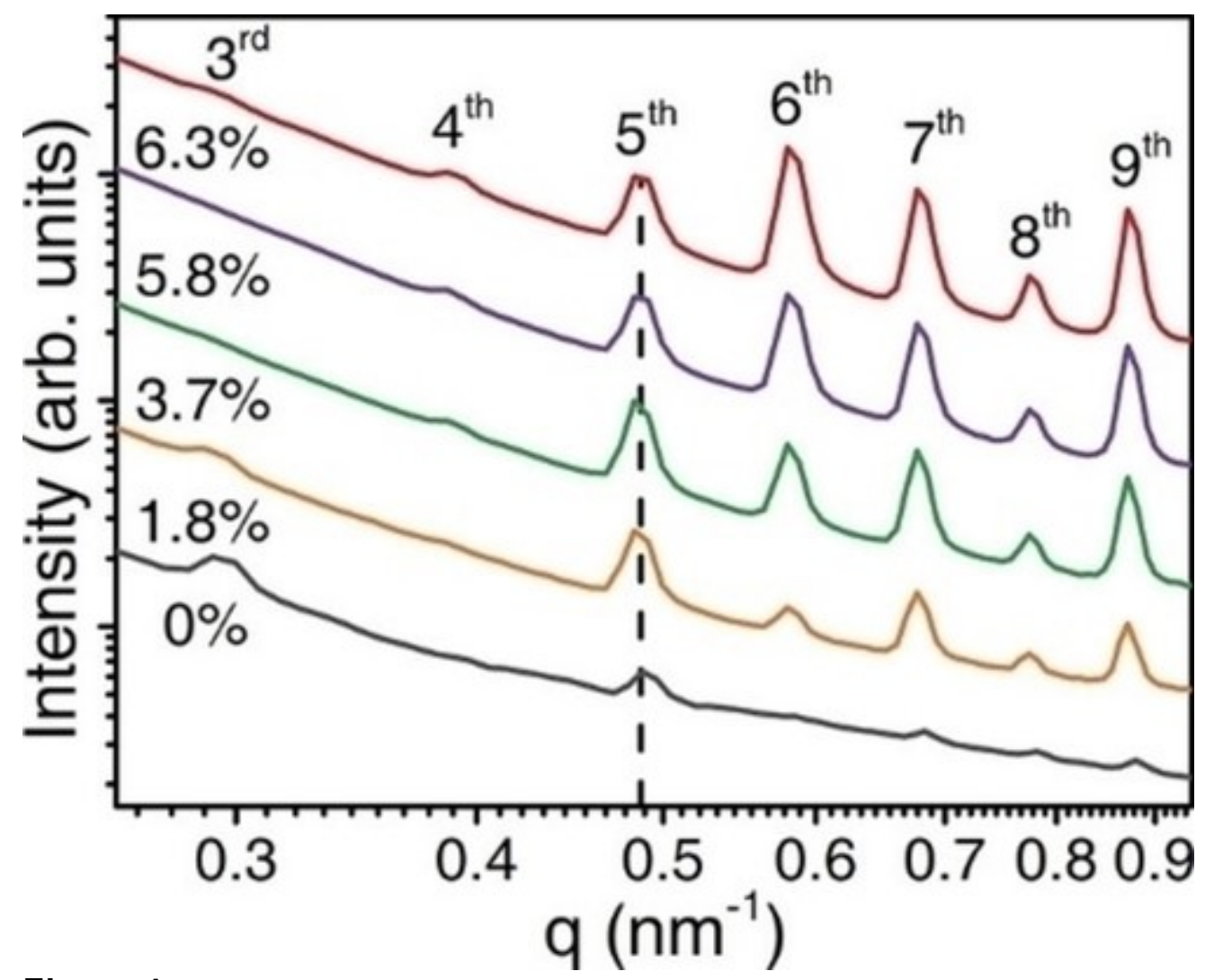

Figure 4

Open in figure viewerPowerPoint

SAXS diffraction patterns for untanned ovine skin $(0 \%)$ and BCS tanned leather with $1.8 \%$, $3.7 \%, 5.8 \%$ and $6.3 \%$ chrome offer. Selected diffraction peaks corresponding to $q=2 \pi n / D$ where $n$ is the peak order and $D$ is the $D$-period, are labelled. Reproduced with permission from Ref. [4r]. Copyright 2018, American Chemical Society.

Prabakar and co-workers investigated the real-time changes in collagen structure using different tanning agents. $\underline{4 p}$ The relative intensity of the fifth and third order peaks were calculated. The relative intensity increased steadily throughout the experiment for BCS, indicating the long-range order and electron density increased with the introduction of $\mathrm{Cr}^{3+}$ into the collagen matrix. With ZIR, there was a sharper increase in intensity in the first 10 mins, indicating the $\mathrm{Zr}^{4+}$ was readily taken into the collagen matrix. The ALS samples showed a lower increase in relative intensity, suggesting that fewer links were being formed. Similar trends were observed when comparing the third order peak to other strong diffraction peaks, except for the sixth order peak which has been correlated with dehydration. $\underline{\mathbf{4 p}}$ 
The relative intensity of the third and second order peaks $\left(R_{3 / 2}\right)$ has been associated with the varied intermolecular structures in the gap/overlap region of collagen which in turn result in differences in its structural resistance to osmotic shrinkage. $\underline{4 \mathrm{~s}}, \underline{24} R_{3 / 2}$ increased significantly on addition of chromium sulphate to hides treated with different pre-treatments, indicating that the fibril contraction during the removal of unbound water was reduced due to the more constrained collagen structure formed during the chromium-collagen reaction. The glutaraldehyde pre-treated samples had higher $R_{3 / 2}$ than the other samples before the addition of chromium, but lower $R_{3 / 2}$ after the addition of chromium, due to the glutaraldehyde initially reacting with the lysine residues of collagen and subsequently minimizing the reaction between the aspartic and glutamic acid residues with chromium. $4 \mathbf{s}$

\subsubsection{Fibril orientation}

Previous studies have attempted to objectively evaluate the collagen orientation in a sample using the scattering of light. $\underline{25}$ Herman's orientation factor was introduced for the characterization of the orientation of polymer chain and has been used to quantify the orientation of collagen fibrils in skin. $\underline{\mathbf{2 6}}$ In 2002, van Zuijlen et al. proposed an orientation index for collagen, using confocal laser-scanning microscopy and Fourier analysis to analyze the collagen orientation of burn scars in human skin. $\underline{27}$

Haverkamp and co-workers studied the orientation of collagen in leather using SAXS analysis by investigating the width of the peaks in azimuthal angle plots of ovine and bovine leather (Figure $\underline{5}) . \underline{4}, \underline{4 e}, \underline{28}$ They later introduced the orientation index (OI), which was defined by the following calculation: $\underline{4 \mathrm{~b}}$

$$
\mathrm{OI}=\frac{90^{\circ}-\mathrm{OA}}{90^{\circ}}
$$




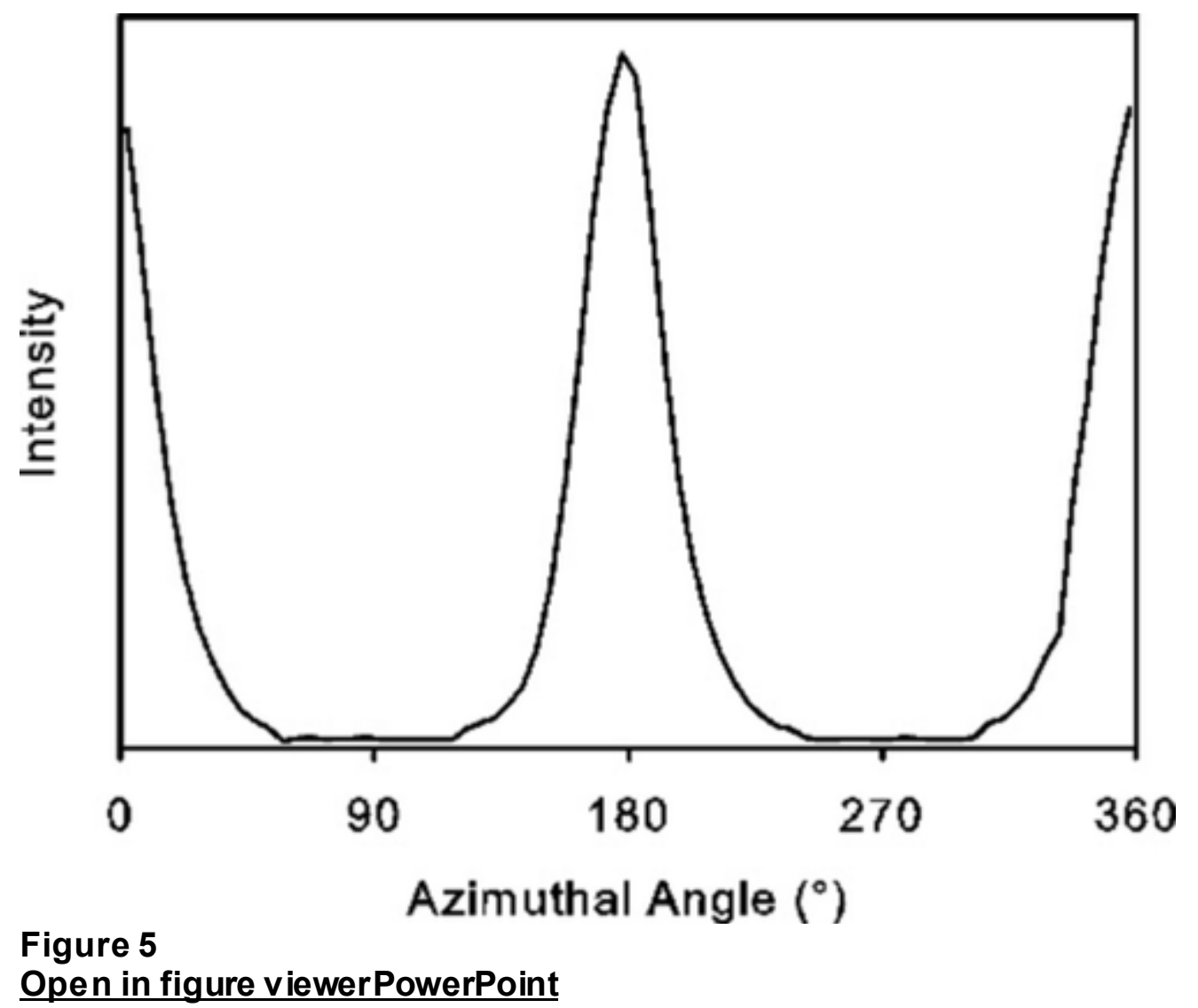

Example of a plot of the intensity variation with azimuthal angle for one collagen peak. Reproduced with permission from Ref. [4e]. Copyright 2013, American Chemical Society. where the $\mathrm{OA}$ represents the orientation angle, the minimum azimuthal angle range centered at $180^{\circ}$ which contains $50 \%$ of the fibrils. The Ol is calculated from the spread in the azimuthal range of the most intense Bragg peak or the peak used to calculate the $D$-period. An OI of 1 indicates the fibrils are oriented parallel to each other, while an OI of 0 indicates the fibrils are randomly oriented. ${ }^{[4 b-j, 41-0,29]}$

This Ol was used to investigate the relationship between collagen orientation and other properties of leather, particularly strength. Strong leathers were observed to have a higher Ol than weak leathers, which suggests highly oriented collagen fibrils give stronger leather. $\underline{\mathbf{4 b}}, \underline{\mathbf{4 c}}$ One study also found a significant correlation between Ol and strength for seven mammal species, including sheep, possum, cattle, goat, water buffalo, horse and deer. $\underline{4 e}$

The OI measurements are directionally dependent and have been taken both normal to the surface (isotropic) and edge-on (cross-sectional) (Figure $\underline{\mathbf{6}}$ ). $\underline{\mathbf{3 0}}$ For the study on seven mammal species, Ol from measurements taken normal to the leather surface were found to be lower than those taken edge-on, and only the edge-on measurements had a significant correlation with tear strength.4e A study on ovine, bovine and cervine leathers found no correlation between strength and OI measurements taken normal to the leather 
surface. $\mathbf{4 g}$ In another study, normal OI measurements and tear strength were not significantly correlated for bovine leather, but both normal and edge-on OI measurements were significantly lower for low-strength ovine leathers compared to high-strength ovine leathers. $\underline{\mathbf{4 c}}$ As the edge-on values have been found to have more significance in relation to the strength of leather, recent studies have focused on edge-on measurements of $\mathrm{OI}$ only. ${ }^{\left[4 h, 4^{\circ}\right]}$

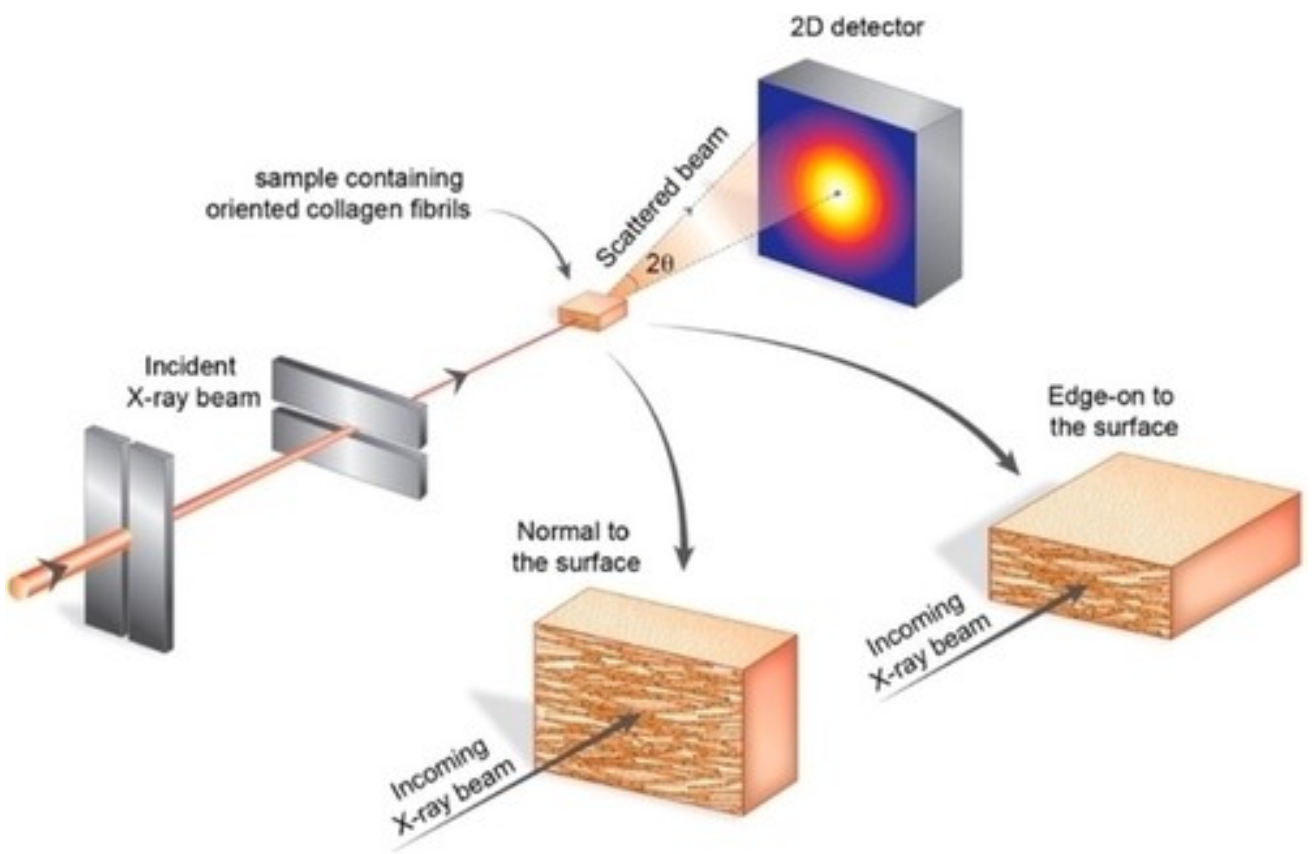

Figure 6

\section{Open in figure viewerPowerPoint}

SAXS experimental set up for measuring the orientation index edge-on and normal to the surface. Reproduced with permission from Ref. [30]. Copyright 2015, American Chemical Society.

The relationships between Ol and the appearance of the grain surface in leather have also been investigated. Looseness is a defect which results in wrinkles and creases in the final leather product.31 SAXS analysis on bovine loose leather determined it had more highly aligned collagen fibrils than tight leather $\underline{4 \mathrm{~m}}$ The OI of loose leather was also fairly uniform throughout the leather cross-section, whereas the tight leather had a more varied OI, with a lower OI of collagen fibrils in the center. Loose leather had a higher tensile strength than tight leather, which was consistent with previous studies showing a correlation between OI and strength. $4 \mathrm{~m}$ Pebble is the characteristic surface pattern of deer leather and is considered to be a desirable feature. ${ }^{\left[4^{\circ}\right]}$ The average OI of the well-pebbled deer leather was found to be slightly higher (but significantly different) than that of the poorly pebbled leat her. Well-pebbled leather had a higher tear strength, which was explained by the overall higher average $\mathrm{Ol}$ and the more even $\mathrm{OI}$ through the cross-section of the leathers. ${ }^{\left[4^{\circ}\right]}$ 
When investigating the relationship between OI and other variables for ovine leat hers at different stages of processing, the varying thickness of the skin during the processing was found to affect the OI. So that the Ol change due to change in thickness could be separated from the Ol change due to other variables.

A calculation was introduced by Sizeland et al. for the change in Ol with thickness changes: $4 \mathrm{j}$

$$
\theta_{2}=\tan ^{-1}\left(\frac{T_{2}}{T_{1}} \tan \theta_{1}\right)
$$

where $T_{1}$ is the original thickness, $T_{2}$ is the new thickness, $\theta_{1}$ is the angle of the collagen fiber to the plane of the leather and $\theta_{2}$ is the newangle of the fiber after the increase in leather thickness.4j Once the leather thickness was taken into account, no correlation was found between Oland $\mathrm{pH} .4 \mathrm{j}$ OI was found to be lower in dry (salted, pretanned, dry crust and dry crust staked) samples than wet (fresh green, pickled, wet blue and retanned) ovine leather samples.4j This adjusted Ol was also used when investigating loose leather during processing. Loose pickled hide and wet blue had a lower OI than tight pickled hide and wet blue.4n The large increase in the Ol of loose leather during processing was thought to be due to the leather swelling, breaking the connections between layers: this is a well-known technological consequence of over swelling, which is not reversible by deliming. $\underline{\mathbf{n}}$

A paper in 2016 investigated the effect of different tanning agents (chromium, zirconium, tetrakis hydroxymethyl phosphonium sulfate (THPS), mimosa and oxazolidine) on the structure of collagen. $\underline{\mathbf{k}}$ Skins tanned with THPS or oxazolidine had higher OI values than those tanned with chromium or zirconium. The leathers with higher shrinkage temperatures were found to be those tanned with chromium or zirconium, which had lower Ol. $\underline{4 k}$ Although the authors suggest that a more crosslinked collagen produced stronger material, with lower $\mathrm{OI}, \underline{\mathbf{4} \mathbf{k}}$ these observations suggest a correlation between Ol and the filling effect of bulkier tanning molecules. There is typically no correlation between leather strength and the outcome of tanning in terms of hydrothermal stability, indeed it is well known in tanning technology that the more we modify the collagen structure, the weaker it becomes.

The Ol of leather has been observed to increase with strain. $\underline{\mathbf{4 f}}, \underline{\mathbf{4 h}}-\underline{\mathbf{4}} \mathbf{j}$ The application of strain during the tanning process has been investigated with the idea of artificially increasing the $\mathrm{OI}$ to give a stronger leather.4d A change in Ol was observed as a result of the strain applied during tanning, and the stretched material was found to have a higher tear strength.4 $\mathbf{d}$ It has been noted that the increase in OI from stretching leather may be due to the reduced sample thickness. $\underline{4 h}$

While the $\mathrm{OI}$ is a convenient way of describing the level of orientation of collagen in leather, a recent study has shown that a significant amount of information about the orientation is lost 
when using a single index.26a Interestingly, the same OI can be calculated for samples with one sharp peak and an isotropic component, one broad peak or multiple peaks in the azimuthal plot, when normalized using the cumulative intensity. $\underline{\mathbf{2 6 a}}$

\subsubsection{Hydration}

Water plays an important role in stabilizing the collagen matrix. $\underline{32}$ The triple helix is surrounded by a cylinder of hydration, a supramolecular matrix, where water molecules hydrogen bond to amino acid residues, particularly hydroxyproline, to create water bridges. 32a, 32b Water also prevents collagen fibers in leather getting within close proximity to form additional chemical bonds which would stiffen the leather and reduce its strength.15 Therefore the hydration level is critical for the quality of the leather.

The intensities of the diffraction peaks of a SAXS spectrum can be used to compare the hydration levels of leather samples. A strong sixth order diffraction peak in comparison with the seventh or eighth order peak has been associated with a dry collagen sample. $\underline{3 a}$ In a later paper, Maxwell et al. noted that the intensities of the fifth and sixth order diffraction peaks gave an indication of the sample hydration. $\underline{3 \mathrm{c}} \mathrm{A}$ strong fifth order diffraction peak indicated high levels of hydration, while a strong sixth order diffraction peak indicated dehydration. $\underline{\mathbf{3 c}}, \underline{10 \mathrm{c}}$

The tanning reaction is affected by the hydration state of the collagen matrix as water occupies regions in the collagen molecule where tanning reactions can take place.15 On chrome tanning, an increase in the relative intensity of the sixth order peak was observed for hide samples. This indicates that the covalent bonding of chromium to collagen resulted in the dehydration of the collagen molecule since there is displacement of the water in the supramolecular matrix by $\mathrm{Cr}^{3+} . \underline{4 \mathbf{s}}$ When tanning ovine skins with different chromium concentrations, the sixth order peak increased with increasing concentration. However, at chromium concentrations greater than $3.7 \%$, the sixth order peak increased at a relatively faster rate, which indicated the increased dehydration of the collagen. This was attributed to the displacement of water due to chromium deposition through non-covalent interactions such as hydrogen bonding. $4 r$ Therefore both covalent and non-covalent interactions of chromium with collagen result in the dehydration of the collagen molecule, with the chromium occupying the space previously held by water molecules.

The effect of pre-treatments on the hydration environment of the collagen by observing changes in the relative intensity of the sixth order peak for equally air-dried samples pretreated with different complexing agents was also studied. $\underline{45}$ The higher $R_{6 / 8}$ peak of GA samples compared to STD samples was attributed to the formation of covalent bonds between GA and amino groups of collagen during fixation. This bonding would reduce the 
number of sites available for water to hydrogen bond with collagen, and hence result in a more dehydrated collagen structure. A decrease in $R_{6 / 8}$ for carboxyl or hydroxyl group containing pre-treatments (SF, DSP and MMT) was observed compared to STD samples. $\underline{4 \mathbf{s}}$

\subsubsection{Axial periodicity ( $D$-period)}

$D$-period, the axial staggering distance of collagen molecules can be calculated from the diffraction peaks on the SAXS spectra, following the same equation used for the intermolecular lateral packing:

$$
d=2 \pi n / q
$$

Several methods have been reported to calculate the $D$-period from SAXS spectra. Some studies determined the $D$-period by fitting a Gaussian curve to the sixth order diffraction peak of an integrated intensity plot and determining the position of the center or maximum of that peak. $\left.{ }^{4 a}, 4 h, 4 j, 4 n, 4^{\circ}, 33\right]$ Others took the central value of several collagen peaks (usually fifth to tenth order), calculated the $D$-period for each using the peak orders and averaged the resulting values. $\underline{\mathbf{4 b}}, \underline{\mathbf{4 c}}, \underline{\mathbf{4 e}}, \underline{\mathbf{4 f}}, \underline{\mathbf{4}}$ Prabakar and co-workers used a program to fit a number of peaks (usually third to ninth order) with the calculated $D$-period being the value that best fits all peaks. $\underline{4 p}-\underline{4 s}$

Analysis of the $D$-period of ovine and bovine leather has found a significant difference between the two, with ovine leather showing a larger $D$-period. $\underline{\mathbf{4 b}}$ Across a sample of ovine leather, three sampling positions were found to have statistically different $D$-periods, with the belly having the highest $D$-period, followed by OSP and then the neck. $\underline{4 \mathrm{c}}$

Multiple studies have observed changes in the $D$-period of the collagen molecules during leather processing. Liming of bovine hide was observed to reduce the $D$-period by around 1 $\mathrm{nm}$, giving the hide a $D$-period similar to that of parchment. $\underline{3 \mathrm{c}} \mathrm{A}$ slight decrease in $D$-period was observed ( $65.5 \mathrm{~nm}$ to $64.5 \mathrm{~nm}$ ) when pickled ovine skins were treated with the pretanning agent Zoldine ZE, a bifunctional oxazolidine. $\underline{\mathbf{4 p}}, \underline{\mathbf{4 q}}$ This was thought to be caused by the reaction of the lysine side chain amino acid groups with the aldehyde groups of Zoldine ZE, forming a Schiff's base adduct which can then react with other amino acid residues. $\underline{4 p}$ Only slight changes in $D$-period were observed after subsequent tanning with BCS, ZIR or ALS, which suggested the terminal amino acid side chain groups had all reacted during the pretanning step (Figure $\underline{7}$ ). $\underline{\mathbf{4} p}$ The results presented are consistent with the differences in filling effects of the three reagents. No further change in $D$-period was observed during the main tanning stage with $\mathrm{BCS}$, sodium silicate (So-Si) and $\mathrm{BCS}+\mathrm{So}-\mathrm{Si}$ after pre-tanning with Zoldine ZE, which was again attributed to the exhaustion of the terminal amino acid residues by the pre-tanning agent. $4 \mathrm{q}$ 


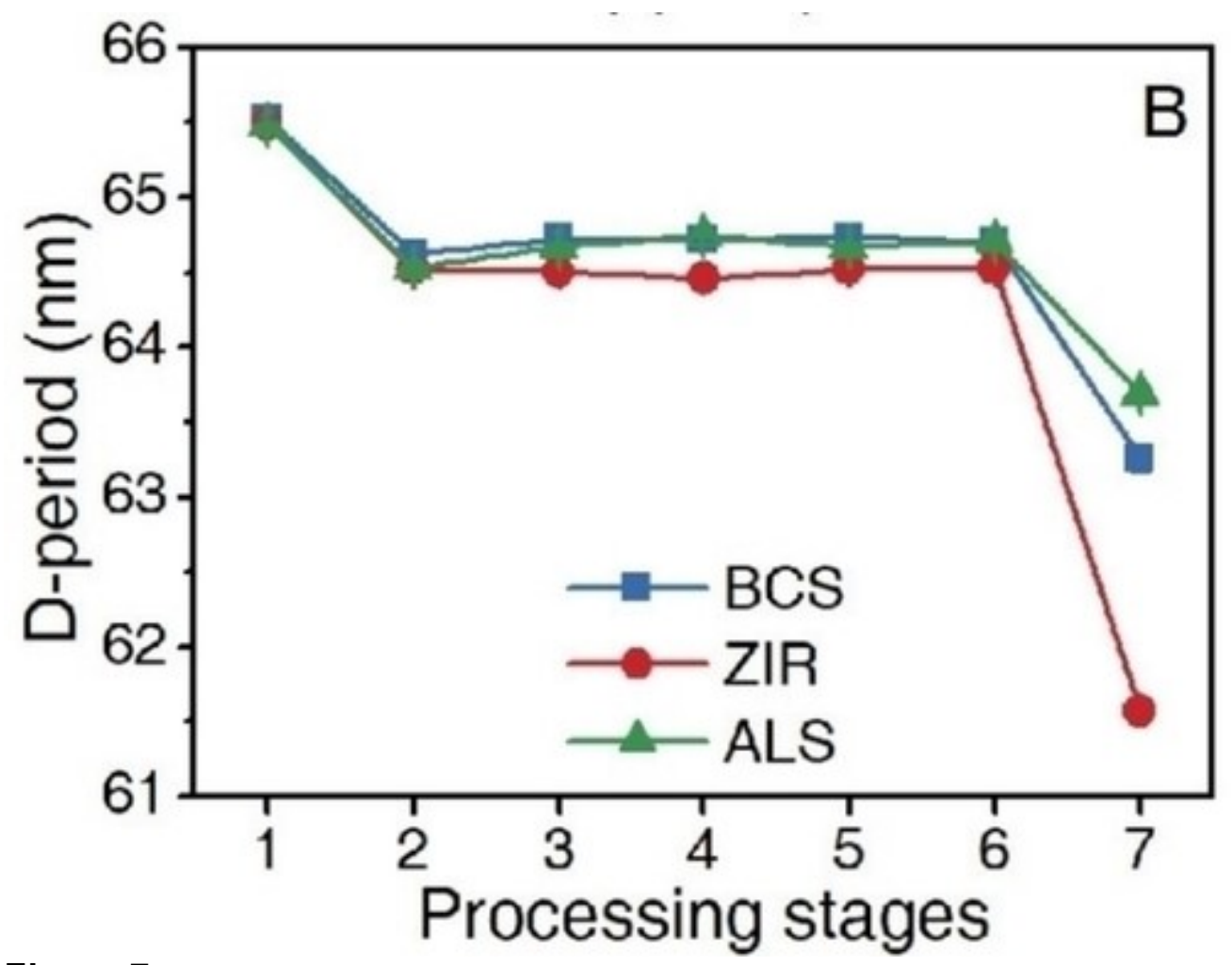

Figure 7

\section{Open in figure viewerPowerPoint}

Changes in D-period after each processing stage: (1) pickled, (2) pretanned, (3) after main tanning, (4) retanned, (5) fat-liquored, (6) wet leather and (7) dry leather. Reproduced with permission from Ref. [4p]. Copyright 2018, American Chemical Society.

In the absence of Zoldine ZE, Maxwell et al. observed an increase in the $D$-period of the collagen molecules of bovine hide upon chrome tanning. $3 \mathbf{b}$ Similarly, Prabakar and coworkers observe an increase in the $D$-period of ovine skins with increasing chromium concentration from $0 \%$ to $1.8 \%$. 4 r However, when the chromium concentration was increased from $1.8 \%$ to $6.3 \%$, no further change in the axial periodicity was observed, which suggested that all telopeptidyl aspartic and glutamic acid residues of collagen were covalently bound to chromium at a concentration of $1.8 \%$. At concentrations above $1.8 \%$, the $D$-period did not change as reactions in this region were no longer possible., $\underline{4} \mathbf{r}$

The $D$-period of collagen can also be affected by the amount of water present in the matrix.2d, 10a, 24, 33, 34 When leather is dried, a large decrease in $D$-period is observed. $\mathbf{4 j}, \underline{\mathbf{4}}, \underline{\mathbf{4 q}}$ The removal of unbound water is known to collapse the collagen structure, greatly reducing the $D$-period. $\underline{\mathbf{4 q}}, \underline{\mathbf{3 5}}$ The decrease in $D$-period upon drying was much larger for ZIR treated leathers than for ALS or BCS (Figure $\underline{7}$ ). In ZIR tanning, the amino side chain groups interact with a combination of cationic, neutral and anionic species by hydrogen bonding and electrostatic interactions. $\underline{\mathbf{4}}, \underline{\mathbf{5 b}}, \underline{\mathbf{1 5}}$ Removal of water from the ZIR tanned leather during drying would result in a greater collapse of the collagen structure due to the predominant hydrogen bonding interactions, resulting in a smaller $D$-period. $\underline{\mathbf{p}}$ 
Moreover, the $D$-period changes were observed to have no direct correlation with the $\mathrm{pH}$ at different stages of leather processing. $\mathbf{4} \mathbf{j}$ it is more likely to be related to the reagents that caused the $\mathrm{pH}$ changes.

Studies have investigated the relationship between $D$-period and strength for ovine and bovine leathers. No correlation was found between $D$-period and strength for ovine leathers. $\underline{\mathbf{4 c}}, \underline{\mathbf{4 e}}$ However, another study found that while there was no correlation for edgeon measurements, normal ovine measurements of weak leather showed a significantly higher $D$-period for the grain compared to the corium. $\underline{\mathbf{4 b}}$ No significant differences were observed for the stronger ovine leather. $\underline{\mathbf{b}}$ Edge-on parallel measurements for bovine leather suggested that increased strength was correlated with increased $D$-period. $\underline{\mathbf{4 b}}$

\subsubsection{Fibril diameter}

Using SAXS to measure the fibril diameter of collagen gives the average collagen fi bril diameter of a scanned area containing a large quantity of fibrils and requires no sample preparation before the analysis. $\underline{4}$

Collagen fibril diameter can be used as a measure of the swelling (water imbibition) of leather. Swelling can occur when the $\mathrm{pH}$ of the surrounding matrix is lower or higher than the isoelectric point of the collagen. $\underline{\mathbf{3 6}}$ Prabakar and co-workers observed an increase in fibril diameter for the skins treated with sodium silicate and attributed it to the high pHconditions $(>11) .4 \mathrm{q}$ This high $\mathrm{pH}$ would result in the penetration of unbound water, and hence swelling, due to a reduction in the intermolecular interactions between collagen molecules. $4 \mathrm{q}$ Maxwell et al. observed a similar increase at high $\mathrm{pH}$ in the average fibril diameter of bovine hide after liming, although the suggested reason in this case was the removal of hyaluron releasing the pressure constraint on the fibrils. $\underline{3 \mathrm{c}}$ Air-drying of leather after treatment resulted in a reduction in fibril diameter, attributed to the collapsed intermolecular lateral packing due to the removal of unbound water. $\underline{\mathbf{4 q}}$

Coating on the outside of the collagen fibrils were also proposed to increase the fibril diameter. ALS treated samples showed a higher percentage increase in fibril diameter than BCS and ZIR treated samples, suggesting the formation of coating by the aluminosilicate/poly(carboxylic acid), $\underline{\mathbf{p}}$ and this was supported by scanning transmission electron microscopy coupled with energy dispersive X-ray spectroscopy (STEM-EDS) analysis. $\underline{4 p}$ ALS leathers also have a plumper feel after drying compared to BCS and ZIR leathers.

The fibril diameters of ovine and bovine leathers were found to be statistically different. 4 I However, no correlation was found between leather strength and collagen fibril diameter when these values were plotted for a variety of animals including crocodile, deer, 


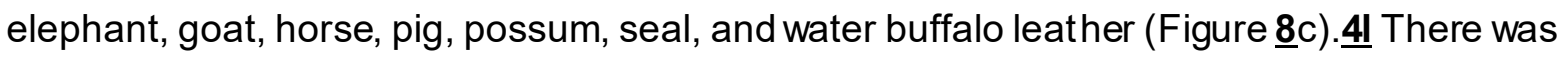
also no significant correlation between strength and fibril diameter for ovine leather samples (Figure $\underline{\mathbf{8}} \mathrm{a}$ ). However, for bovine leather there was a correlation between strength and fibril diameter, with stronger leather consisting of fibrils with larger diameter (Figure $\underline{8} b$ ). $\underline{.1}$
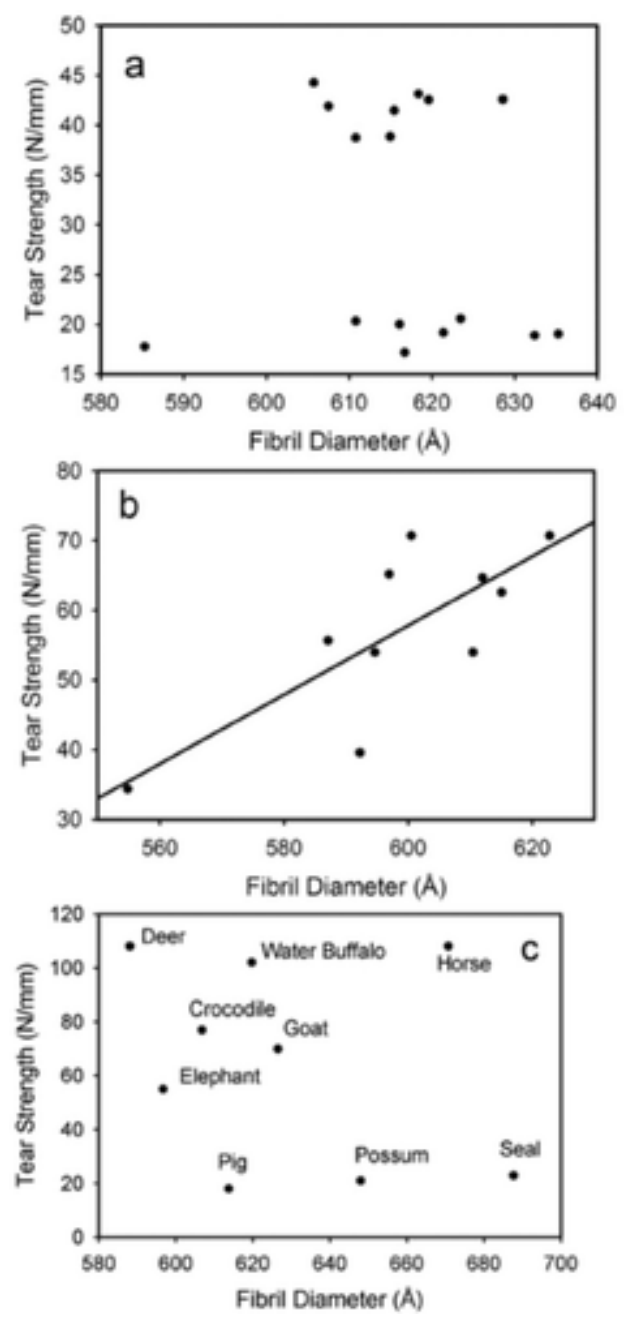

\section{Figure 8}

\section{Open in figure viewerPowerPoint}

Collagen fibril diameter versus tear strength for (a) ovine leather, (b) bovine leather and (c) a range of leathers from other animals, where each point is the average value from 12-20 diffraction patterns. Reproduced with permission from Ref. [4 I]. Copyright 2013, American Chemical Society.

\section{Summary}

X-ray scattering studies of leather at different stages of processing have provided valuable information on the properties of leathers and how they relate to collagen structure at the molecular level. The WAXS spectrum provided understanding of the intermolecular lateral packing, amorphous features and helical rise per residue of leather collagen. From SAXS analysis, information has been gained on chemical fixation, fibril orientation and diameter, hydration and axial periodicity of collagen in leather samples. 


\section{Outlook}

Synchrotron SAXS has played an integral role in collagen structural studies for several years now. Initial X-ray scattering studies of leather focused on understanding how certain features of the collagen structure were linked to the physical and intrinsic properties of leather. While these studies expand on our knowledge of leather structure, the impact on the tanning industry will be minimal as it will be difficult to apply to processing to make leathers of improved quality. More recent studies have focused on SAXS analysis of the tanning process and how chemical fixation works at the molecular level. Completed studies have already shown progress on ways of reducing chrome usage in leather production while retaining leather properties. In future, the X-ray scattering analysis on leather needs to focus on building a more systematic understanding of the collagen structure of leather by connecting the various interpretations that the researchers have achieved so far. It requires more fundamental information about the collagen structure to fill the remaining gaps of knowledge, such as the origin of various peak intensities at different orders, the effects of tanning processes on overall scattering intensity and the changes in the long-range order of collagen by different types of tannages. This would allow researchers to provide detailed predictions of the tanning processes so as to enable minimized chemical usage and guide the development of new tanning agents. Fundamental studies on collagen in leather will help to make a more environment-friendly and economically sustainable future for the leather industry.

\section{Acknowledgements}

The authors would like to thank the Ministry of Business, Innovation and Employment (MBIE) for funding through grant LSRX-1801 and LSRX-1701.

\section{Conflict of interest}

The authors declare no conflict of interest.

\section{References}

\section{Biographical Information}

Jenna Buchanan has been working in Dr Sujay Prabakar's research group at the Leather and Shoe Research Association of New Zealand in Palmerston North since 2018. Her research is focused on developing novel crosslinking agents to replace or minimize chrome in leather processing. She received her BSc in chemistry and statistics in 2015 and her MSc in chemistry in 2017 from Massey University in Palmerston North. 


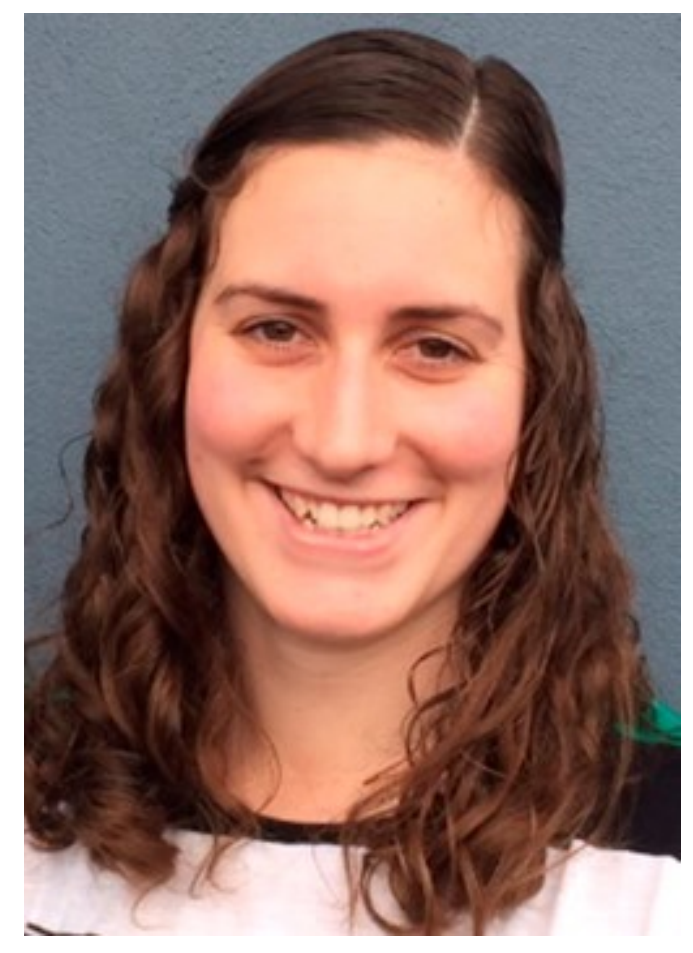

\section{Biographical Information}

Yi Zhang received his B.S. in chemistry from Fudan University in 2014 and his B.S. (Hons) from the University of Auckland in 2015. Since 2016, he has worked in Dr Sujay Prabakar's group at the Leather and Shoe Research Association of New Zealand, studying collagen structure using synchrotron SAXS.

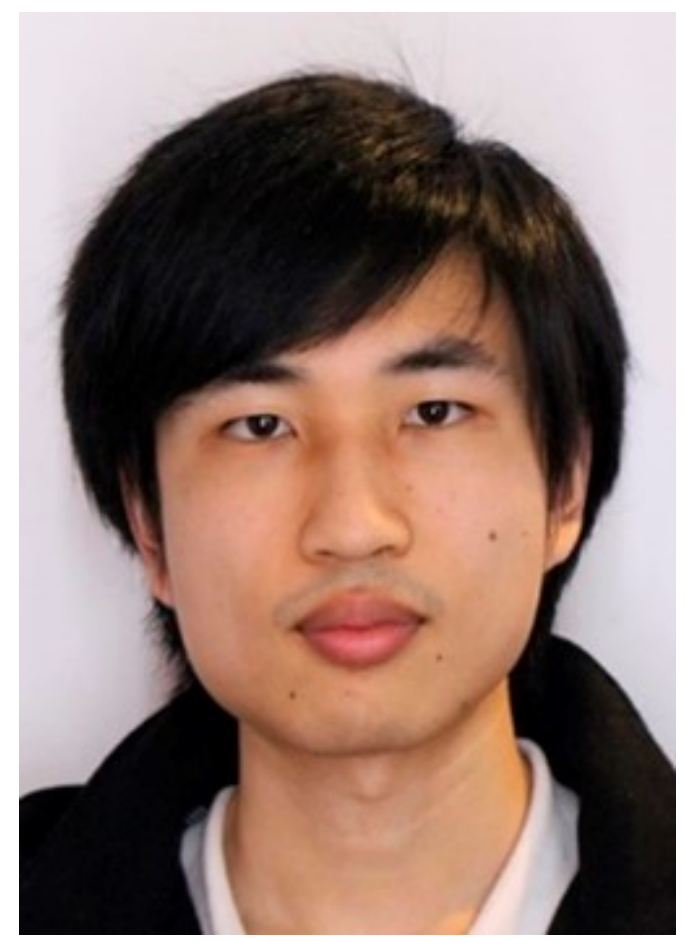

Biographical Information 
Geoff Holmes is a leather technologist who obtained his Degree in Applied Science from Kingston University in 1986 and HND in Leather Technology from Northampton

Leathersellers' Centre, UK, in 1989. He has over 30 years'experience in the leather industry in various production and technical roles, the last 14 years involved in research at the Leather and Shoe Research Association of New Zealand (LASRA). He is the current LASRA director.

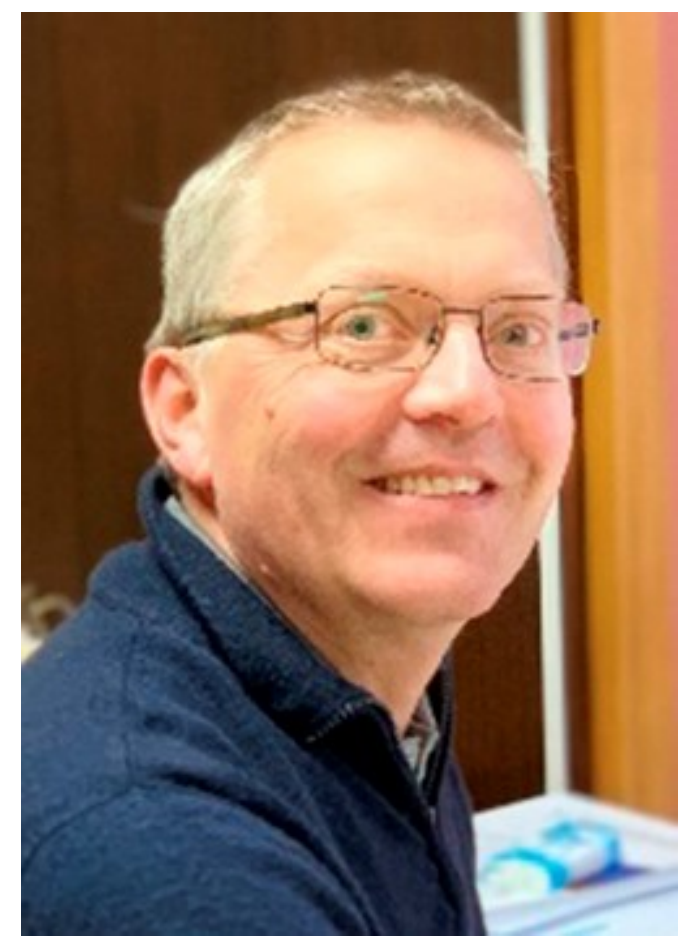

\section{Biographical Information}

Anthony Covington is Emeritus Professor of Leather Science at the University of Northampton, UK. He has been engaged in the global leather industry for over 40 years, undertaking research and development and teaching, specializing in tanning theory, process development and clean technology. He is the author of over 300 technical publications, including one book, which have received over 1500 citations in the literature. He gained his $\mathrm{PhD}$ in physical organic chemistry at Stirling University, followed by post-doctoral research in physical chemistry at the University of Newcastle upon Tyne. 


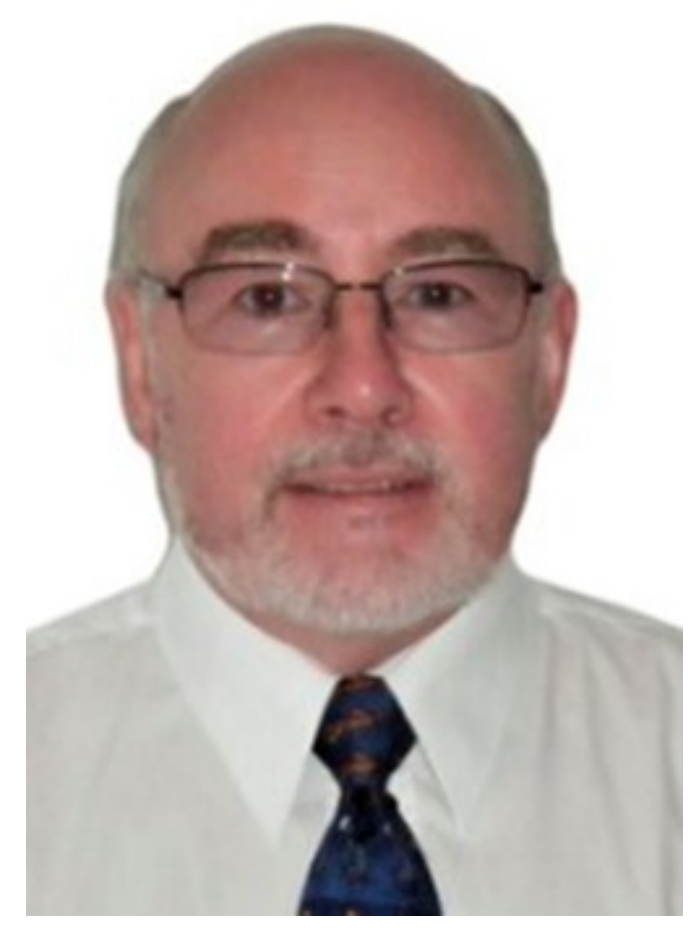

\section{Biographical Information}

Sujay Prabakar is a scientist at the Leather and Shoe Research Association of New Zealand based at Palmerston North. He studied for his Ph.D. in the School of Chemical \& Physical Sciences, Victoria University of Wellington, NZ. At present, he leads an interdisciplinary materials chemistry research team studying molecular levels changes in collagen during cross-linking and biomaterials for drug delivery.

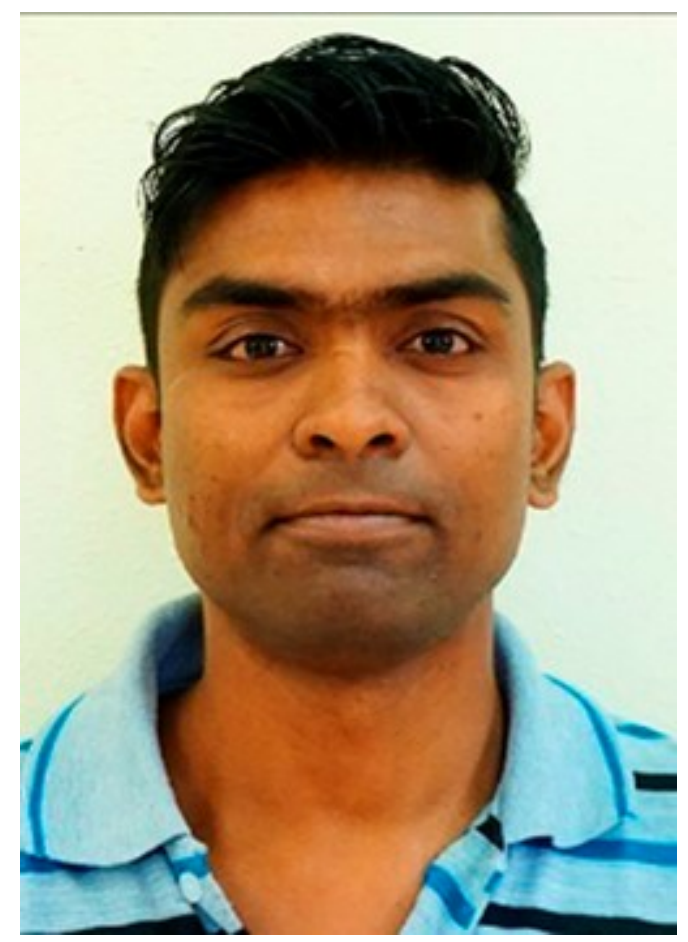

